\title{
Cost Effectiveness of Triplet Selinexor-Bortezomib-Dexamethasone (XVd) in Previously Treated Multiple Myeloma (MM) Based on Results from the Phase III BOSTON Trial
}

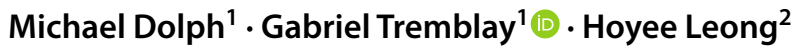

Accepted: 13 July 2021 / Published online: 9 August 2021

(c) The Author(s) 2021

\begin{abstract}
Background and Objective Nearly all patients with multiple myeloma undergo multiple rounds of therapy. The phase III BOSTON trial of once-weekly selinexor and once-weekly bortezomib with dexamethasone (XVd) vs twice-weekly bortezomib and dexamethasone $(\mathrm{Vd})$ is the basis for this cost-effectiveness analysis in previously treated multiple myeloma from a US commercial payer perspective over a lifetime horizon.

Methods A partitioned survival model enabled use of direct overall survival and progression-free survival curves from BOSTON to generate four health states for $\mathrm{XVd}$ and $\mathrm{Vd}$ : progression-free survival on treatment, progression-free survival off treatment, post-progression, and mortality. Using a 1-week cycle length, benefits and costs were discounted at 3.0\% annually. Additional comparators were included in an exploratory analysis that compared XVd against seven additional regimens (six triplets, one doublet).

Results After considering costs, utility, progression, and survival, the base-case incremental cost-effectiveness ratio of XVd vs Vd was $\$ 475,430$ /quality-adjusted life-year (QALY). The 50\% cost-effectiveness probability midpoint was near $\$ 470,000 / Q A L Y$, based on a probabilistic sensitivity analysis. The robustness of the analysis was supported by additional scenario assessment and deterministic and probabilistic sensitivity analyses, which generally demonstrated little variance, with greatest sensitivity to variations in discount rates and utility values. In an exploratory analysis against external comparators, XVd showed a higher QALY gain with a lower cost (i.e., dominance) compared with lenalidomide/dexamethasone (Rd), pomalidomide/bortezomib/dexamethasone (PVd), and carfilzomib/pomalidomide/dexamethasone (KPd).

Conclusions Addition of XVd to the previously treated multiple myeloma treatment landscape provides a novel oral treatment option, which, when compared to Vd in the base-case analysis resulted in an incremental cost-effectiveness ratio of $\$ 475,430 /$ QALY. Exploratory analyses comparing against external comparators suggest that XVd was dominant vs Rd, PVd, and KPd.
\end{abstract}

\section{Plain Language Summary}

Patients with multiple myeloma often relapse and require multiple treatments to extend survival while maintaining quality of life. Many of the standard treatment regimens include twice-weekly bortezomib, which is associated with potentially severe peripheral neuropathy. The novel triplet regimen of once-weekly selinexor and once-weekly bortezomib with dexamethasone $(\mathrm{XVd})$ improves cancer response and progression-free survival while decreasing the rate of peripheral neuropathy. This study used economic modeling to calculate the cost of the triplet XVd regimen per life-year gained and per quality-adjusted life-year gained. XVd had a lower cost with a higher quality-adjusted life-year benefit compared with lenalidomide/dexamethasone, pomalidomide/bortezomib/dexamethasone, and carfilzomib/pomalidomide/dexamethasone in previously treated multiple myeloma.

Gabriel Tremblay

gabrieltremblay@pshta.com

1 Purple Squirrel Economics Montreal, 1600 Notre Dame W, Suite 201, Montreal, QC H3J 1M1, Canada

2 Karyopharm Therapeutics, Newton, MA, USA

\section{Introduction}

Multiple myeloma (MM) is the second most common hematologic malignancy, representing $1 \%$ of all cancers and $2 \%$ of all cancer deaths with approximately 34,920 new MM cases and 12,410 deaths anticipated in 2021 in the USA 


\section{Key Points for Decision Makers}

For patients with previously treated multiple myeloma, once-weekly oral selinexor/bortezomib/dexamethasone $(\mathrm{XVd})$ reduces rates of peripheral neuropathy and increases survival by 0.33 life-years and 0.34 qualityadjusted life-years vs twice-weekly bortezomib/dexamethasone $(\mathrm{Vd})$.

$\mathrm{XVd}$ is associated with a lifetime incremental total cost increase of $\$ 159,557$ USD, driven by higher drug costs and partially offset by lower subsequent therapy, medical, and mortality costs vs Vd.

$\mathrm{XVd}$ is dominant compared to lenalidomide/dexamethasone (Rd), pomalidomide/bortezomib/dexamethasone (PVd), and carfilzomib/pomalidomide/dexamethasone (KPd) in exploratory analyses.

[1]. Despite the approval of several novel agents in recent years, MM largely remains incurable and fatal, with nearly all patients relapsing after each sequential therapy and ultimately developing progressive refractory disease. In addition to its clinical and quality-of-life impacts, MM carries a significant economic burden [2]. Novel therapies have increased survival, but they have also been associated with increased medical costs, particularly for injectable treatments, with overall costs increasing in later lines of therapy [3-5].

Bortezomib has been used since 2003 in a variety of MM treatment regimens [6, 7]. Most regimens for previously treated MM utilize twice-weekly bortezomib, administered in the clinic, which can be burdensome for patients, caregivers, and the healthcare system. Moreover, twiceweekly bortezomib induces significant peripheral neuropathy (PN), with reported rates of $35-55 \%$ in doublet and triplet regimens, often leading to treatment interruptions, dose reductions, treatment discontinuation, and ultimately, poorer patient outcomes [8-11]. Peripheral neuropathy can be minimized with once-weekly bortezomib therapy, and many physicians employ once-weekly bortezomib-based regimens despite a lack of clinical data supporting them [12]. Compared to twice-weekly bortezomib regimens for previously treated MM, new regimens effectively utilizing once-weekly bortezomib are expected to offer benefits such as the convenience of fewer clinic visits, reduced PNassociated treatment-emergent adverse events (TEAEs) from bortezomib, improved progression-free survival (PFS), and a better overall response rate $[8,10,13,14]$.
Selinexor (Xpovio ${ }^{\mathrm{TM}}$; Karyopharm Therapeutics, Inc., Newton, MA, USA) is an oral, first-in-class, selective inhibitor of nuclear export that works synergistically with proteasome inhibitors (such as bortezomib) and dexamethasone to selectively kill malignant plasma cells [15]. Selinexor is approved by the US Food and Drug Administration in combination with bortezomib and dexamethasone for the treatment of adults with MM who have received at least one prior therapy. Its original approval was in combination with low-dose dexamethasone for the treatment of adults with previously treated MM who have received at least four prior therapies and whose disease is refractory to at least two proteasome inhibitors, two immunomodulatory drugs, and an anti-CD38 monoclonal antibody [15].

A phase III clinical trial, BOSTON (NCT03110562), compared once-weekly selinexor and once-weekly bortezomib in combination with low-dose dexamethasone ( $\mathrm{XVd}$ ) to the standard twice-weekly bortezomib and moderate-dose dexamethasone $(\mathrm{Vd})$ regimen [16]. In addition to improvement in the primary endpoint of PFS and secondary efficacy endpoints such as overall response rate and duration of response across patient subgroups (including patients with a high cytogenetic risk and those $\geq 65$ years of age), $\mathrm{XVd}$ demonstrated improvements in health-related quality-oflife outcomes, while using $40 \%$ less bortezomib and $25 \%$ less dexamethasone during the first 24 weeks of treatment. Patients receiving XVd reported significantly lower sensory symptom scores on the European Organization for Research and Treatment of Cancer QLQ-CIPN20 scale, with a trend towards reduced motor symptom scores, and persistent reductions in pain as reported in the European Organization for Research and Treatment of Cancer QLQ-C30, likely due to lower levels of bortezomib-induced PN with the lower bortezomib dose in XVd [17]. The BOSTON trial allowed patients in the $\mathrm{Vd}$ arm to cross over to a selinexor-containing regimen upon objectively confirmed MM progression; nonetheless, there was a trend towards improved overall survival (OS) in the XVd arm. This analysis evaluated the cost effectiveness of XVd vs Vd in previously treated MM from a US commercial perspective with additional administration costs included, using patient-level data from BOSTON [18], which formed the basis for the clinical trial publication [16]. The model also evaluated the cost effectiveness of $\mathrm{XVd}$ relative to seven comparator regimens: daratumumab/ pomalidomide/dexamethasone (DPd), daratumumab/lenalidomide/dexamethasone (DRd), lenalidomide/dexamethasone (Rd), pomalidomide/bortezomib/dexamethasone (PVd), elotuzumab/pomalidomide/dexamethasone (EPd), carfilzomib/ pomalidomide/dexamethasone (KPd), and daratumumab/ bortezomib/dexamethasone (DVd). 


\section{Methods}

\subsection{Target Population and Subgroups}

The target population was adults with previously treated MM. At model onset, the average patient age was 67 years, based on the mean age of patients at initiation in BOSTON. While the base-case analysis of the model assumed that all MM treatment lines were included, additional scenarios were tested in which the population was limited to certain treatment line subgroups (second line only vs third line or higher). The base-case population was based on the proportion of second-line and third-line or higher patients in BOSTON (49.3\% and 50.7\%, respectively).

\subsection{Treatments, Comparators, Cycle Length}

The main treatment under economic evaluation was $\mathrm{XVd}$. Selinexor and dexamethasone are both administered orally, and while bortezomib is available in several forms, it was assumed to be administered subcutaneously based on current usage. The main comparator was $\mathrm{Vd}$, as per the BOSTON trial. A 1-week model cycle length was used to provide sufficient granularity to capture all relevant costs and outcomes.

\subsection{Perspective, Time Horizon, Discount Rate}

The base-case analysis was conducted from a US commercial payer perspective (including additional administrationrelated costs) using a lifetime horizon (assumed to be 40 years, after which virtually all patients would be deceased), which enabled the capture of all potential long-term costs and benefits. A 3\% annual discount rate was used for both benefits and costs, as per the Second Panel on Cost-Effectiveness in Health and Medicine [19]. All cost inputs were obtained from commercial sources, when possible. In the absence of commercial payer values, US Centers for Medicare \& Medicaid Services costs were used as a proxy. A breakdown of model inputs can be found in the Electronic Supplementary Material (ESM).

\subsection{Conceptual Framework and Model Structure}

The cost-effectiveness analysis (CEA) was structured in Microsoft Excel using a partition survival model based directly on the clinical trial OS and PFS data [20]. Survival curves were extrapolated over the entire model horizon (lifetime) based on parametric extrapolations, with the most appropriate functional forms selected based on statistical fit and clinical robustness. The model considers the amount of time spent in each of four health states:
- (1) Progression-free (PF) [on treatment]: patients were assumed to initiate treatment with $\mathrm{XVd}$ or $\mathrm{Vd}$ at the beginning of the model. These patients were at risk of disease progression or death and could also discontinue treatment before disease progression.

- (2) PF (off treatment): patients were PF but have discontinued primary therapy. As with health state (1), these patients could move to the post-progression (PP) or mortality health states.

- (3) PP: patients were at risk of death and can receive further treatment lines in the model. Patients were assumed to remain in this health state until mortality. In PP, patients would incur lower utility and higher medical costs relative to the pre-progression states.

- (4) Mortality: mortality was determined by the BOSTON OS data, extrapolated over a lifetime horizon.

The base-case model compared the cost effectiveness of $\mathrm{XVd}$ vs Vd. Additional analyses compared XVd to other doublet and triplet regimens in an exploratory analysis, as there are very few published US-based economic models comparing triplet regimens in previously treated MM (Figs. 1 and 2).

\subsection{OS}

Overall survival in the base-case model was based on the BOSTON data and calculated from the date of randomization to the date of death. Patients without events were censored at the date of study discontinuation or date of last participating visit, whichever occurred first. Note that BOSTON allowed Vd patients to cross over to XVd after progression. To accurately capture the true OS benefit of Vd, extrapolation curves were fitted to regression-adjusted OS data, which included a covariate for crossover. The Kaplan-Meier OS curve and the extrapolated long-term OS curves for the XVd and $\mathrm{Vd}$ arms are displayed in Fig. 3. The blue lines represent the direct trial data (Kaplan-Meier curves), while the red lines indicate the extrapolated data. Based on the assessment of statistical fit and clinical expectations, OS extrapolations for both $\mathrm{XVd}$ and $\mathrm{Vd}$ were based on parametric curves using exponential functional forms.

In the BOSTON trial, median OS for patients in the $\mathrm{Vd}$ arm was 25.0 months at a median follow-up of 17.5 months (note that the Kaplan-Meier curve for Vd only presents noncrossover patients, as crossover adjustment was applied to Vd patients), while median OS in the XVd arm was not reached at a median follow-up of 17.3 months. Following crossover adjustment and extrapolation, median OS for Vd patients was 36.6 months. At the time of this analysis, median OS for XVd was not reached, and $24.1 \%$ of patients on XVd and $30.0 \%$ of patients on Vd had died. Following extrapolation, median OS for XVd was 40.1 months. 
Twelve-month OS in the model was $81.3 \%$ and $79.7 \%$ for $\mathrm{XVd}$ and $\mathrm{Vd}$, respectively.

\subsection{PFS}

Progression-free survival for the base-case model was also based directly on the BOSTON data and was calculated from the date of randomization until the first date of confirmed disease progression as per International Myeloma Working Group response criteria, or death due to any cause,

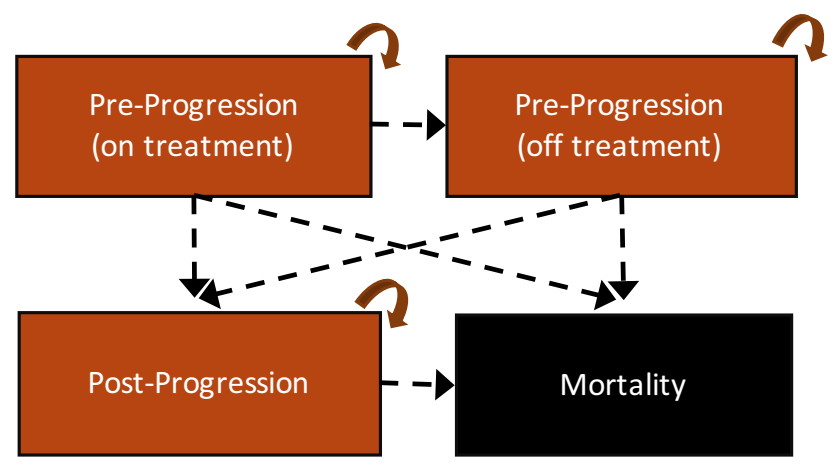

Fig. 1 Health state diagram. Curved arrows represent patients remaining in the same health state; dashed arrows represent patients progressing to different health states whichever occurred first. The Kaplan-Meier PFS curve and the extrapolated long-term PFS curves for the $\mathrm{XVd}$ and $\mathrm{Vd}$ arms are displayed in Fig. 4. With OS, PFS extrapolation curve selection was based on the assessment of statistical fit and the most likely reflection of clinical expectation. Therefore, parametric curves using log-normal functional forms were used for both $\mathrm{XVd}$ and $\mathrm{Vd}$.

The XVd arm showed significant improvement vs Vd for PFS, with a median of 13.9 months vs 9.5 months ( $p=$ 0.0075), i.e., $47 \%$ improvement in median PFS. After extrapolation, the median PFS for XVd and Vd was 13.9 and 9.0 months, respectively. Twelve-month PFS in the model was $54.9 \%$ and $40.8 \%$, and average time in the PFS state was 29.5 months and 19.6 months for XVd and Vd, respectively.

\subsection{Treatment Duration}

Durations of primary therapy for both $\mathrm{XVd}$ and $\mathrm{Vd}$ were based on the time to treatment discontinuation data from BOSTON (i.e., treatment discontinuation and PFS were extrapolated separately in the model, which allowed both endpoints to be based directly on the trial data). After discontinuation, patients were assumed to received secondary therapy for a specified duration of 4 months. Patient-level data were unavailable for the exploratory additional comparator analysis, thus treatment durations were based on the

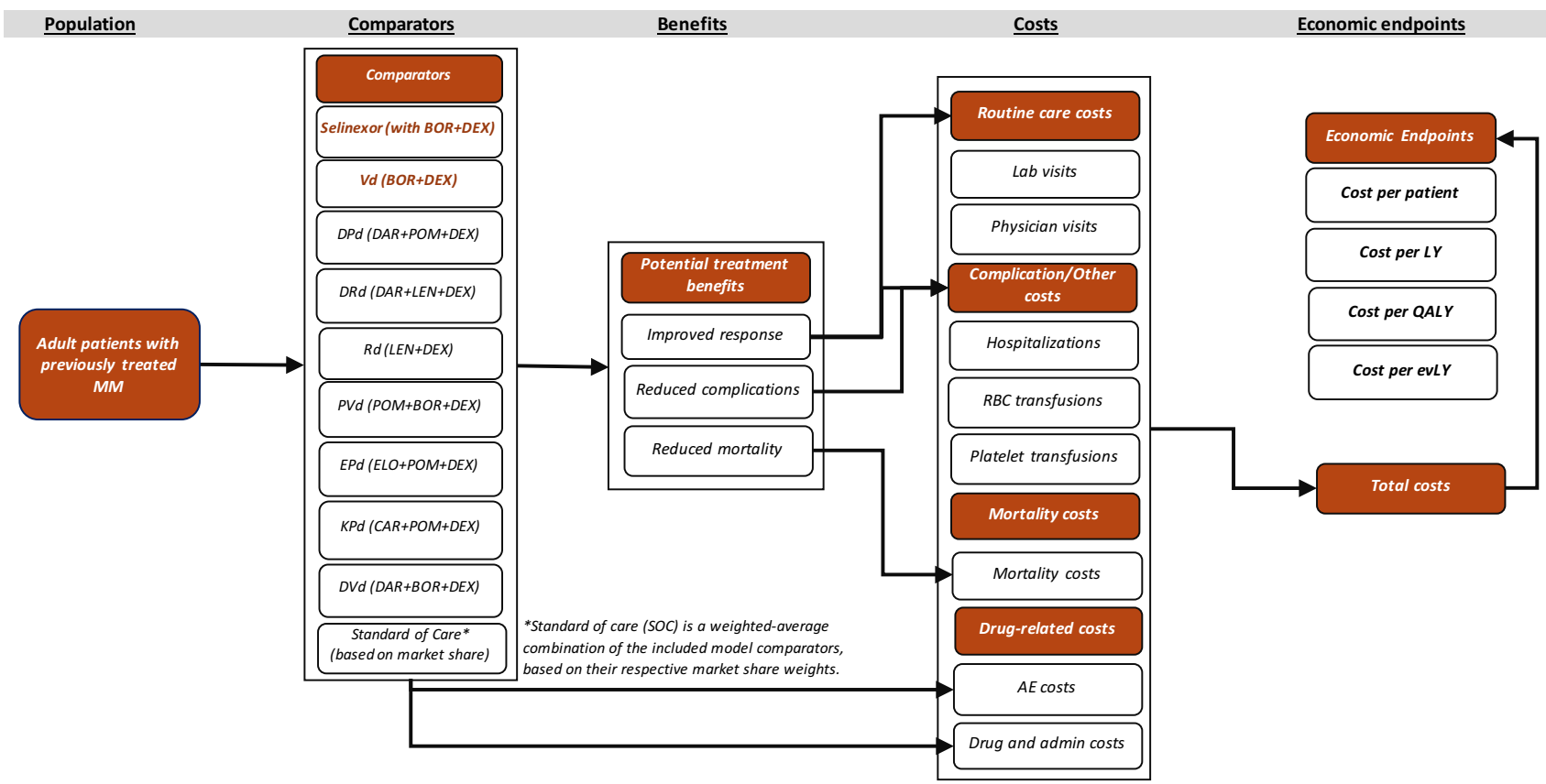

Fig. 2 Conceptual framework. admin administration, $A E$ adverse event, $B O R$ bortezomib, $e v L Y$ equal value life-year, $C A R$ carfilzomib, $D A R$ daratumumab, $D E X$ dexamethasone, $D P d$ daratumumab-pomalidomide-dexamethasone, $D R d$ daratumumab-lenalidomide-dexamethasone, $D V d$ daratumumab-bortezomib-dexamethasone, ELO elotuzumab, EPd elotuzumab-pomalidomide-dexamethasone, $K P d$ carfilzomib-pomalidomide-dexamethasone, Lab laboratory, $L E N$ lenalidomide, $L Y$ cost per life-year, $M M$ multiple myeloma, $P O M$ pomalidomide, $\quad P V d$ pomalidomide-bortezomib-dexamethasone, $R B C$ red blood cell, $R d$ lenalidomide-dexamethasone, $Q A L Y$ qualityadjusted life-year, TEAE treatment-emergent adverse event, $V d$ bortezomib-dexamethasone 
exponentially extrapolated median time until discontinuation (i.e., the point at which $50 \%$ of patients discontinued therapy).

\subsection{Costs}

The following cost categories were included in the model: (1) primary therapy and administration, (2) secondary therapy, (3) routine medical care, (4) TEAEs, and (5) mortality. When necessary, all costs were inflated to 2020 US dollars using an inflation rate of $2.03 \%$, the disease-specific rate for neoplastic diseases [21].

\subsubsection{Drug and Administration Costs}

Both primary and secondary therapy drug costs included wastage (i.e., assuming that any unused drug in a vial will be discarded and calculated on a per-administration basis). The administration cost of oral medication was assumed to be zero, while administration costs of subcutaneous and intravenous treatments were $\$ 80.12$ and $\$ 142.55$ per dose [22].

The average patient weight and body surface area used for weight or body surface area-based dosing therapies were $73 \mathrm{~kg}$ and $1.83 \mathrm{~m}^{2}$, respectively [23,24]. XVd and $\mathrm{Vd}$ doses were adjusted to accurately reflect the relative dose intensity received in BOSTON ( $80 \%$ for selinexor, $90 \%$ for bortezomib, and $91 \%$ for dexamethasone for XVd-treated patients; $99 \%$ for bortezomib and $95 \%$ for dexamethasone for Vd-treated patients, based on median doses received in BOSTON compared with the prescribed doses).

All drug prices were based on the commercial drug prices obtained from Analy\$ource [25]. The weekly dosing and costs of XVd and Vd are displayed in the ESM and were based on the BOSTON clinical trial dosing schedule, which utilizes a 35-day treatment cycle for XVd and 21-day cycles for the first eight treatment cycles followed by 35-day cycles for $\mathrm{Vd}$.

Considering the drug and administration costs as well as the dose reduction experienced in BOSTON, the total drug cost of XVd was $\$ 5619 /$ week while the cost of Vd was $\$ 1754 /$ week. The standard of care cost (applied for secondary therapy) was $\$ 5958$ and was based on the combined market share weighting of therapies. Secondary therapy market shares were assumed to be the same, regardless of a patient's primary therapy received (XVd or Vd). Weekly cost and market share data on the primary and secondary therapy options are available in the ESM.

\subsubsection{Adverse Event Costs}

The model included grade 3+ TEAEs. As low-grade TEAEs do not often incur a significant cost, their associated costs were considered negligible in the model. TEAEs were costed based on a micro-costing approach, where it was assumed that a proportion of TEAEs required inpatient care while the remaining events required outpatient care. The cost of an outpatient visit (\$52.33) was based on the Current Procedural Terminology cost of a physician visit [26], while inpatient costs were extracted from HCUPnet for each specific TEAE based on its respective International Statistical Classification of Diseases and Related Health Problems, 10th Revision code and ranged from $\$ 4634$ to $\$ 13,197$ with a median cost of $\$ 7232$ [27]. The proportion of TEAEs that required inpatient care was assumed to be $4.7 \%$, based on the proportion of all TEAEs in BOSTON that were severe or greater. The incidences of TEAEs for XVd and Vd were sourced from BOSTON, while rates for other included therapies were extracted from their respective clinical trials.

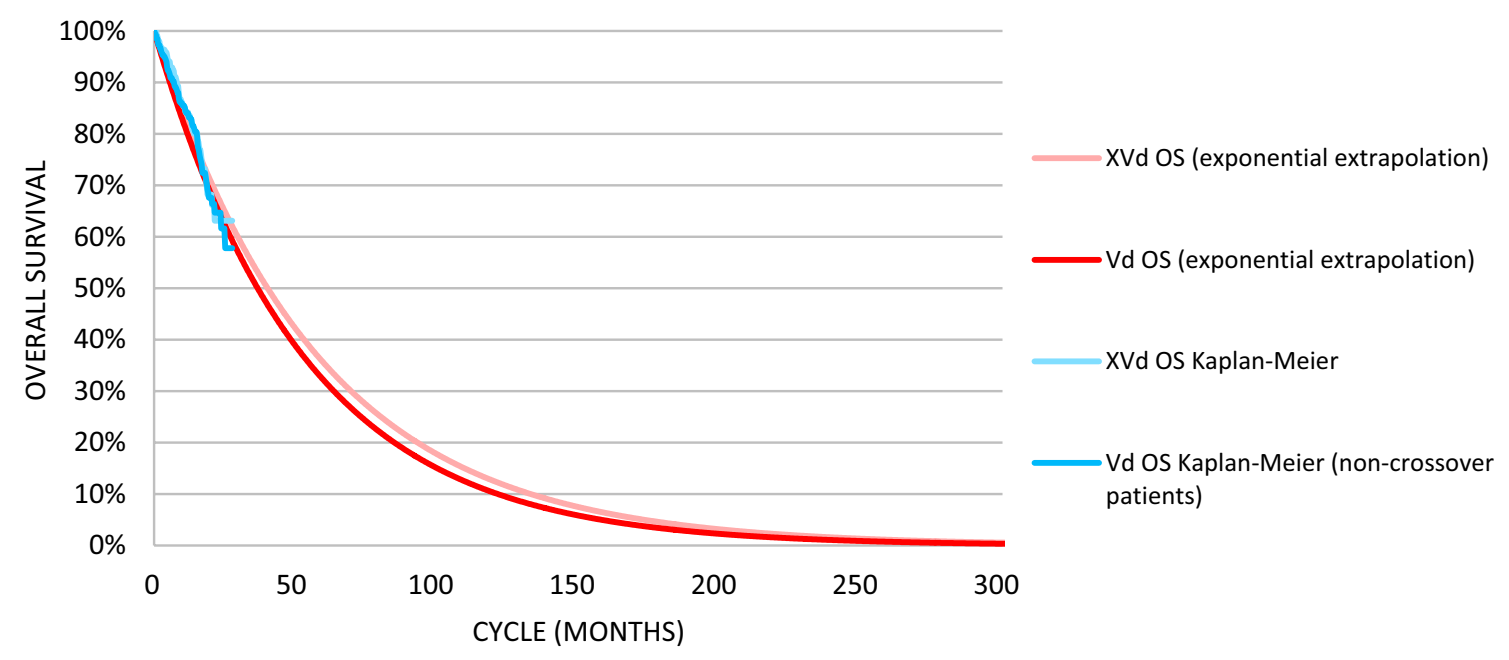

Fig. 3 Long-term overall survival (OS) and Kaplan-Meier curves. Vd bortezomib-dexamethasone, XVd selinexor-bortezomib-dexamethasone. Source: [18] 


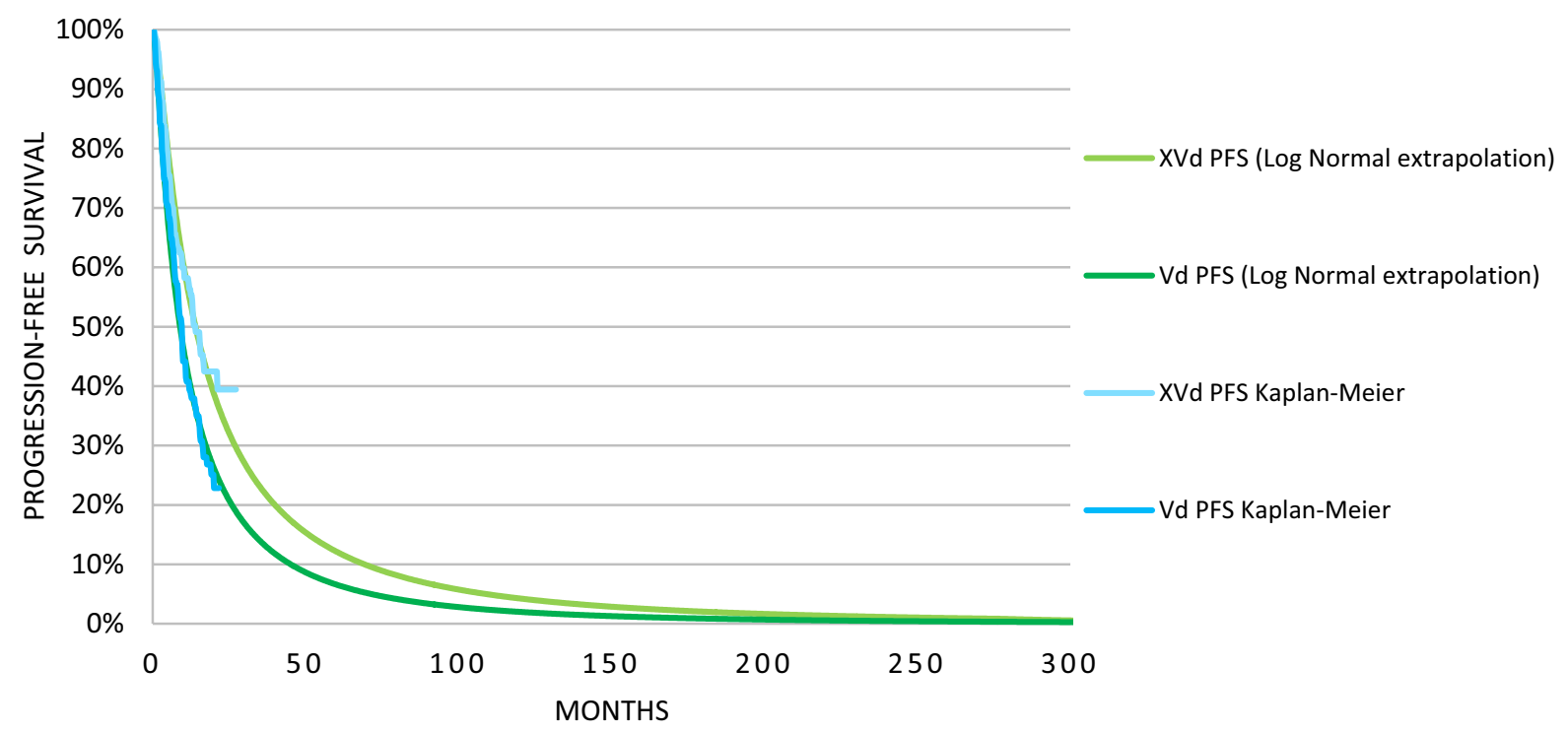

Fig. 4 Long-term progression-free survival (PFS) and Kaplan-Meier curves. $V d$ bortezomib-dexamethasone, $X V d$ selinexor-bortezomib-dexamethasone. Source: [18]

Rates were transformed to 1-week cycle lengths using the probability-to-rate formula. The three most frequent grade 3+ TEAEs for XVd were 'thrombocytopenia' (39.5\%), 'anemia' (15.9\%), and 'pneumonia' (13.8\%) while the three most frequent grade $3+$ TEAEs for Vd were 'thrombocytopenia' (17.2\%), 'pneumonia' (11.8\%), and 'anemia' (10.3\%). The total cost per weekly cycle of TEAEs for $\mathrm{XVd}$ and $\mathrm{Vd}$ were $\$ 9.81$ and $\$ 4.40$, respectively. The average cyclical prevalence and cost of TEAEs for each primary and secondary regimen are presented in the ESM. The average cost per cycle of secondary therapy was $\$ 8.78$.

\subsubsection{Routine Care Costs}

Utilization and unit costs of relevant routine medical resources used are dictated in this section for the PF and PP health states. Routine medical care items included hematologist visits, full blood counts, biochemistry laboratory tests, immunoglobulin tests, protein electrophoresis, urinary light chain excretion, red blood cell transfusions, and platelet transfusions [28]. Resource use frequencies were based on the UK National Institute for Health and Care Excellence technology appraisal for pomalidomide for relapsed/refractory MM, as referenced in [28]. Unit costs were obtained from the US Centers for Medicare \& Medicaid Services based on the relevant Current Procedural Terminology codes for all items excluding the transfusion costs [26]. Transfusion costs were based on published estimates [28].

To derive the final per-cycle costs of routine medical care for the PF and PP health states, annual frequencies were adjusted to 1 -week cycles, which were then multiplied by respective unit costs (Table 1$)$. Weekly routine medical care costs for the PF and PP health states were $\$ 52.15$ and $\$ 56.65$, respectively.

\subsubsection{Mortality}

The inflated end-of-life cost (applied as a one-time cost at time of death) was $\$ 42,702$, obtained from HCUPnet using the code "C90.02 Multiple myeloma in relapse" [30]. Mortality was estimated in the model based on the OS curves from BOSTON and extrapolated over a lifetime horizon.

\subsection{Utility}

Utility values (Table 2) were obtained from the relevant literature assessing utilities using EQ-5D-5L data [31]. In addition to adjusting for TEAE disutility, the final utility values also accounted for an additional 0.17 utility benefit incurred by patients actively achieving a response [29]; this was applied to the proportion of complete responders in each treatment arm (7.2\% for XVd and $4.3 \%$ for Vd). The final utility values used in the model, which include both TEAE disutility and additional response utility, were 0.819 for $\mathrm{XVd}$ and 0.816 for $\mathrm{Vd}$ in the PF health state, respectively, while using the PP utility of 0.638 .

Additional sources of utility values were obtained from the BOSTON clinical trial EQ-5D-5L data and from external literature, which were explored in additional scenario analyses. The BOSTON clinical trial utilities were not used as the base-case values, as the BOSTON data showed a very minimal difference between the PF and PP health states $(0.80$ for 
PF and 0.78 for PP), which likely does not capture the true expected detriment of progressive disease. While the exact reasons for this result are unknown, the utility similarities between health states in the BOSTON data may be due to the rapid sample size erosion of the patients with progressed disease, which may have consisted of a disproportionate number of patients with more severe disease than those progressed patients who remained in the dataset (resulting in an over-estimate of the PP utility). Additionally, interaction effects between time and response status may confound the health state utilities generated by the BOSTON data, further limiting its internal validity.

For these reasons, the base-case analysis used utilities obtained from van Agthoven et al. [31]. While this study had several key differences compared with the BOSTON trial population (most notably being based on UK population tariffs using data from newly diagnosed MM), the utility distinction between health states was considered more representative than the utility values from the BOSTON utility analysis, which showed virtually no difference between the $\mathrm{PF}$ and PP health states.

Disutility scores were based on the BOSTON patientlevel data (EQ-5D-5L) utility analysis and adjusted for age, sex, baseline Eastern Cooperative Oncology Group performance status, and duration of diagnosis. TEAEs without EQ-5D-5L data available in BOSTON were assumed to have disutility equal to the average of all grade 3/4 TEAEs $(-0.0387)$. The average duration of each TEAE was also based on BOSTON patient-level data and applied to the respective disutility and yearly TEAE prevalence estimates to generate the total average disutility for each therapy arm.

Disutility values were applied based on the per-cycle incidences of TEAEs and are displayed in the ESM. The four TEAE categories assumed to produce the greatest disutility include 'fatigue' $(-0.1003)$, 'peripheral neuropathy' $(-0.0582)$, 'anemia' (-0.0558), and 'asthenia' $(-0.0537)$. The total yearly adjusted disutility values associated with $\mathrm{XVd}, \mathrm{Vd}$, and secondary therapy (standard of care) were $-0.003,-0.001$, and -0.002 , respectively.

\subsection{Economic Endpoints}

Four economic endpoints were included in the analysis: cost per patient, cost per life-year (LY), quality-adjusted LY (QALY), and equal value LY (evLY). The methods to calculate cost per LY, QALY, and evLY are similar where the incremental cost of therapy is divided by the incremental benefit [19]. As per the Institute for Clinical and Economic Review's 2020-23 Value Assessment Framework, evLYs value all gains in LYs at a constant utility weight (0.851), such that regardless of age, disability, or illness, all LY gains are valued equally [32]. This approach is aimed at reducing potential discrimination against certain groups (elderly, disabled) by assigning lower values to their lives than others when the QALY is used. However, the QALY was still the primary outcome used in this analysis.

\subsection{Assessment of Uncertainty and Model Validation}

Uncertainty was evaluated using additional scenario analyses, one-way (deterministic) sensitivity analyses, and probabilistic sensitivity analyses (PSAs). The deterministic sensitivity analyses and PSAs use the confidence interval of each input (upper and lower bound estimates) as the new 'varied' input in each scenario, when available. When data were unavailable, a $10 \%$ proxy variation for non-cost-related inputs was used, while a $20 \%$ proxy for medical cost-related inputs was used (based on the large regional and setting variations in medical costs in the USA). To calculate PSAs, all inputs subject to any degree of uncertainty included probability distributions from which a random value could be drawn. Values were randomly selected along each distribution for all inputs simultaneously, once per simulation. The incremental cost-effectiveness ratio (ICER) was noted for each simulation of the CEA. A total of 1000 simulations were undertaken to determine parametric uncertainty and to estimate the probability that the ICER would fall under the varying willingness-to-pay thresholds.

The model underwent quality-control validation and was carefully evaluated to follow the ICER's proposed methodology and international recommendations based on the joint International Society for Pharmacoeconomics and Outcomes Research and Society for Medical Decision Making Modeling Good Research Practices Task Force [33]. Face validity was ensured through clinical and health economic expert guidance throughout the development of the model from the conceptual stage until the finalization of the core model. Additionally, regarding technical validity, the model was subjected to a systematic examination of calculations and Visual Basic for Applications coding accuracy by modeling experts internally and with external reviewers. A costeffectiveness model verification checklist guided the quality-control process, which, among other checks, included extreme value analysis and tracing of calculations. Comments and identified issues were addressed in the final model version. Cross-validation was performed via an assessment of observed PFS and OS outcomes, against which model outputs were compared. These showed good consistency and results were in line with expectations. Finally, regarding external validation, results and model approaches (health states, treatment pathway assumptions, routine care) were compared with previously published relevant economic analyses where available. Additionally, where external data were required to inform model inputs, sources specific to the intervention and population of interest were used. 


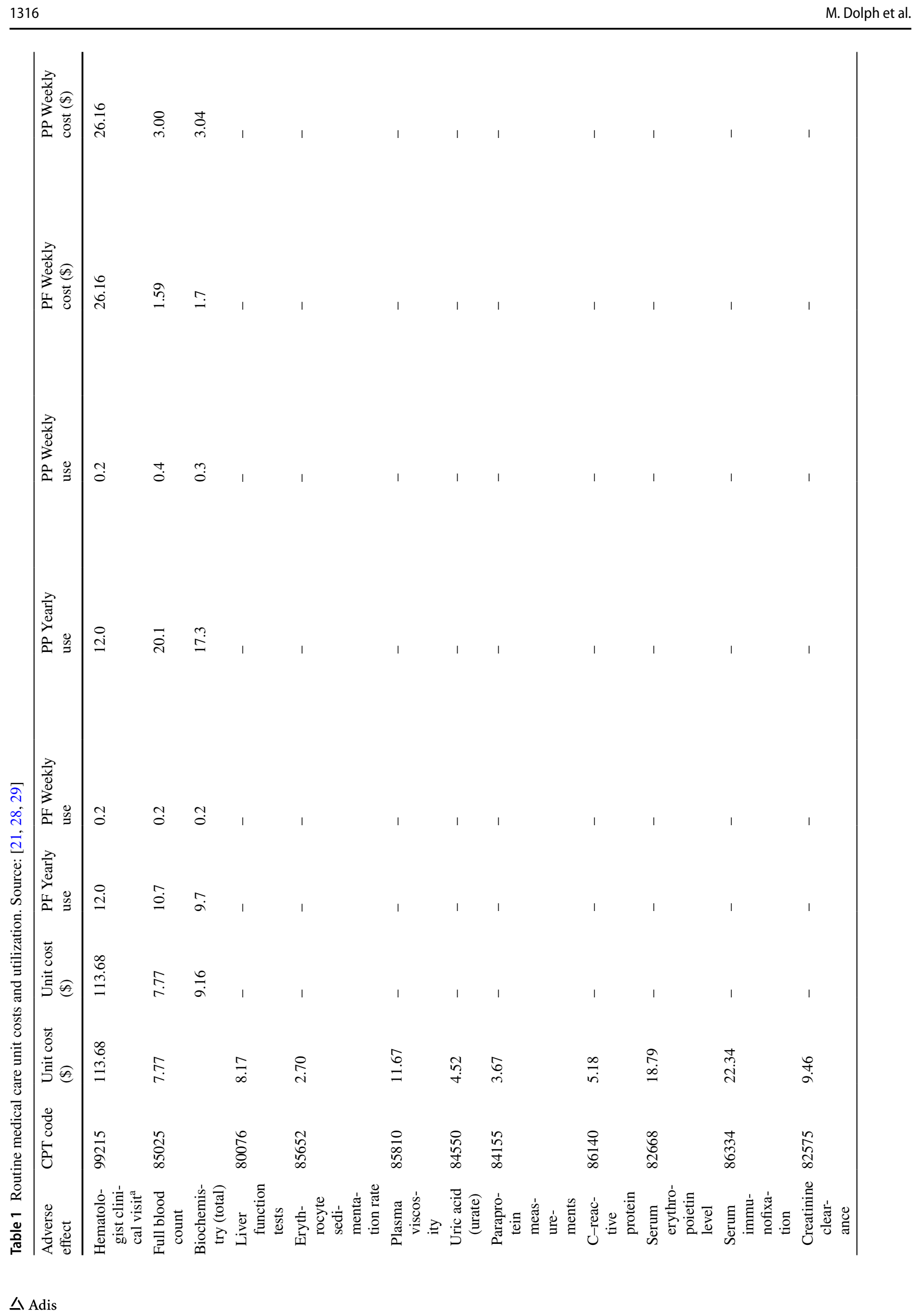




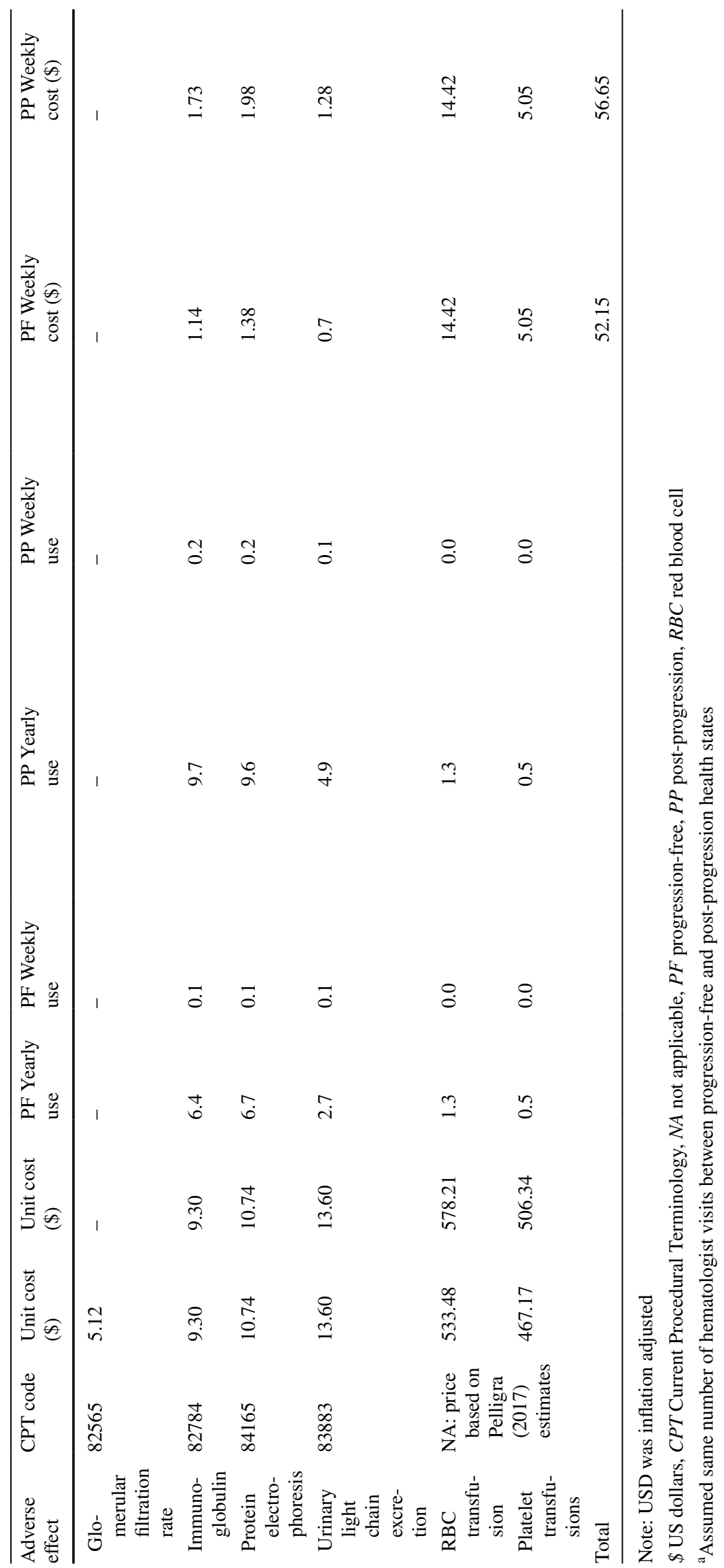


Table 2 Health state utilities. Source: [31]

\begin{tabular}{lll}
\hline Health state & $\mathrm{XVd}$ & $\mathrm{Vd}$ \\
\hline Utility data selected (excluding disutility) & & \\
PF & 0.810 & 0.810 \\
PP & 0.640 & 0.640 \\
Utility data selected (including disutility) & & \\
PF & 0.807 & 0.809 \\
PP & 0.638 & 0.638 \\
Used in model (including disutility and & & \\
responder benefit) & & \\
PF & 0.819 & 0.816 \\
PP & 0.638 & \\
\hline
\end{tabular}

$P F$ progression-free, $P P$ post-progression, $V d$ bortezomib-dexamethasone, $X V d$ selinexor-bortezomib-dexamethasone

\subsection{Comparator Analysis}

While the base-case model was a within-trial model comparing XVd to Vd using extrapolated data directly from the BOSTON trial, exploratory analyses were performed to assess the cost effectiveness of XVd against other important comparators in previously treated $\mathrm{MM}$, as there are very few economic studies comparing other available regimens. By varying which regimen was compared to $\mathrm{XVd}$, the direct costs of the comparator (drug costs and TEAE costs), as well as the efficacy (OS and PFS) changed based on which regimen was selected. The OS and PFS hazard ratios (HRs) used for each comparator were primarily based on a Bayesian network meta-analysis, which was stratified by treatment line (second line vs third line and beyond). All included studies in the network meta-analysis were assessed in a feasibility assessment and were determined to be sufficiently comparable in terms of trial design and baseline characteristics to be included in the analysis. However, HR values by treatment line were limited for some of the included comparators, in which case different statistical adjustment techniques and/or assumptions were required (summarized in the ESM). These assumptions varied based on the availability of the treatment line-stratified HRs for each comparator, where less available data typically required greater assumptions. The final comparator analysis assessed $\mathrm{XVd}$ against each regimen using a "mixed" population that included both second and third or further line patients, derived by calculating weighted average HRs for the mixed population based on the proportion of second and of third or further line patients in BOSTON (49.3\% and 50.7\%, respectively). The final HRs (comparator vs Vd) were applied to the Vd PFS and OS curves to derive the comparator PFS and OS curves.

\section{Results}

\subsection{Incremental Costs and Outcomes}

$\mathrm{XVd}$ in previously treated MM was associated with an incremental total cost increase of $\$ 159,557$ USD over a lifetime horizon compared to $\mathrm{Vd}$. The higher overall cost of XVd vs $\mathrm{Vd}$ was mainly driven by higher primary therapy costs, but was partially offset by lower PP medical costs and mortality costs, and was minimally offset by lower secondary therapy costs (Table 3).

$\mathrm{XVd}$ was associated with an incremental increase of $0.33 \mathrm{LYs}, 0.34$ QALYs, and 0.28 evLYs saved compared to Vd over a lifetime horizon (Table 4). When combining the differences in costs and efficacy gain, the ICERs for cost per LY, QALY, and evLY saved in USD were $\$ 486,533$, $\$ 475,430$, and $\$ 571,719$, respectively.

\subsection{Additional Scenarios}

Additional scenarios were tested where one or multiple inputs were varied at one timepoint to assess scenario uncertainty. Results were most sensitive to changes in extrapolation functional forms (not shown in the graph, as ICERs varied from - \$3.4 to \$27.7 million), variations in time horizon, including/excluding crossover adjustment, and utility source (Fig. 5).

\subsection{Deterministic Sensitivity Analyses}

Deterministic sensitivity scenarios were also conducted, where one input was varied in one direction while all other inputs were held constant. Results were most sensitive to changes in discount rates, and utility values (Fig. 6).

\subsection{PSAs}

PSAs were also conducted (Table 5). Probabilistic results showed an average ICER of $\$ 475,965$ per QALY, with simulations being equally spread among incremental cost ( $y$-axis) and incremental QALY ( $x$-axis) (Fig. 7).

The proportion of simulations falling under the $\$ 150,000$ per QALY, \$300,000 per QALY, and \$500,000 per QALY thresholds were $0 \%, 0 \%$, and $70 \%$, respectively.

The net benefit approach demonstrates the proportion of iterations that were cost effective for XVd and Vd over various ICERs (Fig. 8). The 50\% cost-effectiveness probability midpoint was equivalent to the ICER of the base-case analysis and was near $\$ 470,000$ per QALY. 
Table 3 Discounted costs over a lifetime horizon (USD)

\begin{tabular}{lrrc}
\hline Costs & XVd (USD) & Vd (USD) & Difference (USD) \\
\hline Drug costs & & & \\
Primary therapy & 227,627 & 68,599 & 159,028 \\
Secondary therapy & 104,227 & 104,312 & -85 \\
Adverse event costs & 551 & 326 & 225 \\
Routine medical care costs & & \\
PF medical costs & 5945 & 4053 & 1892 \\
PP medical costs & 6003 & 7092 & -1089 \\
Mortality costs & 37,355 & 37,769 & -414 \\
Total & 381,708 & 222,151 & 159,557 \\
\hline
\end{tabular}

$P F$ progression-free, $P P$ post-progression, $V d$ bortezomib-dexamethasone, $X V d$ selinexor-bortezomib-dexamethasone, USD US dollars

Table 4 Efficacy per therapy

\begin{tabular}{lccc}
\hline Endpoint $^{\mathrm{a}}$ & $\mathrm{XVd}$ & $\mathrm{Vd}$ & Difference \\
\hline LYs (total) & $\mathbf{4 . 2 3}$ & $\mathbf{3 . 9 0}$ & $\mathbf{0 . 3 3}$ \\
LYs (pre-progression) & 2.20 & 1.50 & 0.70 \\
LYs (PP) & 2.03 & 2.40 & -0.37 \\
QALYs (total) & $\mathbf{3 . 0 9}$ & $\mathbf{2 . 7 6}$ & $\mathbf{0 . 3 4}$ \\
QALYs (pre-progression) & 1.80 & 1.23 & 0.57 \\
QALYs (PP) & 1.29 & 1.53 & -0.24 \\
evLYs (total) & $\mathbf{3 . 6 0}$ & $\mathbf{3 . 3 2}$ & $\mathbf{0 . 2 8}$ \\
\hline
\end{tabular}

$e v L Y$ equal value life-year, $L Y$ life-year, $P P$ post-progression, $Q A L Y$ quality-adjusted life-year, $V d$ bortezomib-dexamethasone, $X V d$ selinexor-bortezomib-dexamethasone

${ }^{a}$ Values were discounted

\subsection{Comparator Analysis}

As mentioned, the base-case analysis compared XVd with $\mathrm{Vd}$ and resulted in a cost per QALY of $\$ 475,430$. The basecase ICER (XVd vs Vd) was in the "incremental" quadrant (where XVd has a higher cost but also a higher QALY gain compared with the comparator). Exploratory analyses were also conducted, which compared XVd against other available regimens. When compared with Rd, PVd, and KPd, XVd was shown to be dominant (i.e., having a lower cost and a higher QALY gain vs the comparator). When compared against the remaining regimens (DPd, DRd, EPd, DVd, and standard of care), XVd was "decremental" (i.e., had a lower cost but also a lower QALY gain vs the comparator) (Fig. 9). The ESM details comparator OS and PFS HRs, as well as costs, QALYs, and ICERs. It should be noted that the availability of data used to map the HRs for some of these included additional regimens were limited, and thus several limiting assumptions were required in order to include them in the comparison (described further in the discussion section).

\section{Discussion}

\subsection{Summary of Model Findings}

After considering costs, utility, progression, and survival, the base-case ICER of XVd vs Vd was calculated to be $\$ 486,533$ per LY and $\$ 475,430$ per QALY. The $50 \%$ costeffectiveness probability midpoint was equivalent to the ICER of the base-case analysis and was near $\$ 470,000$ per QALY, based on the PSA. The robustness of the analysis was supported by deterministic sensitivity analyses and PSAs, which generally demonstrated little variance, with greatest sensitivity to variations in discount rates and utility values. The variance of other inputs had relatively smaller impacts on the results. Additional scenario analyses showed greatest sensitivity to changes in extrapolation functional forms, variations in time horizon, including/excluding crossover adjustment, and utility source. The results of this CEA are consistent with those of an independently reported study that calculated an ICER of $\$ 479,572$ per QALY for XVd vs Vd [34].

$\mathrm{XVd}$ provides clear patient benefits, in terms of efficacy and safety, particularly in relation to the reduced dosing frequency of bortezomib, consequent reduction in PN incidence, and positive impact on health-related quality of life compared with $\mathrm{Vd}$. The incremental costs of these benefits are comparable to what has been seen across various novel regimens in previously treated MM [35]. In a recent USfocused CEA, a network meta-analysis was used to compare a standard doublet, Rd, with a series of novel doublet and triplet regimens. The ICER for second-line regimens ranged from about $\$ 51,000 / \mathrm{QALY}$ to $>\$ 450,000 / \mathrm{QALY}$; in the third line, the ICER ranged from over $\$ 60,000 / \mathrm{QALY}$ to over $\$ 500,000 / \mathrm{QALY}$ [36]. Other US-based CEAs in previously treated MM found ICERs from about $\$ 156,000 /$ QALY for daratumumab monotherapy vs pomalidomide [37] to $>\$ 1,300,000 / \mathrm{QALY}$ when comparing DRd to Rd [38]. Thus, the ICER identified in this study for XVd, while high, reflects the high drug costs seen in most novel oncology regimens. Moreover, in this analysis, $\mathrm{XVd}$ was shown to be dominant, with a lower cost and a higher QALY gain, compared with the Rd, PVd, and KPd regimens. XVd represents an option that offers patients efficacy, safety, and healthrelated quality-of-life benefits while reducing bortezomib dosing and allowing for home-based oral administration.

\subsection{Limitations}

The base-case model presented in this document, while comprehensive, had several limitations to note. First, because the utilities derived from BOSTON patient-level data showed very little difference between the $\mathrm{PF}$ and $\mathrm{PP}$ health states 


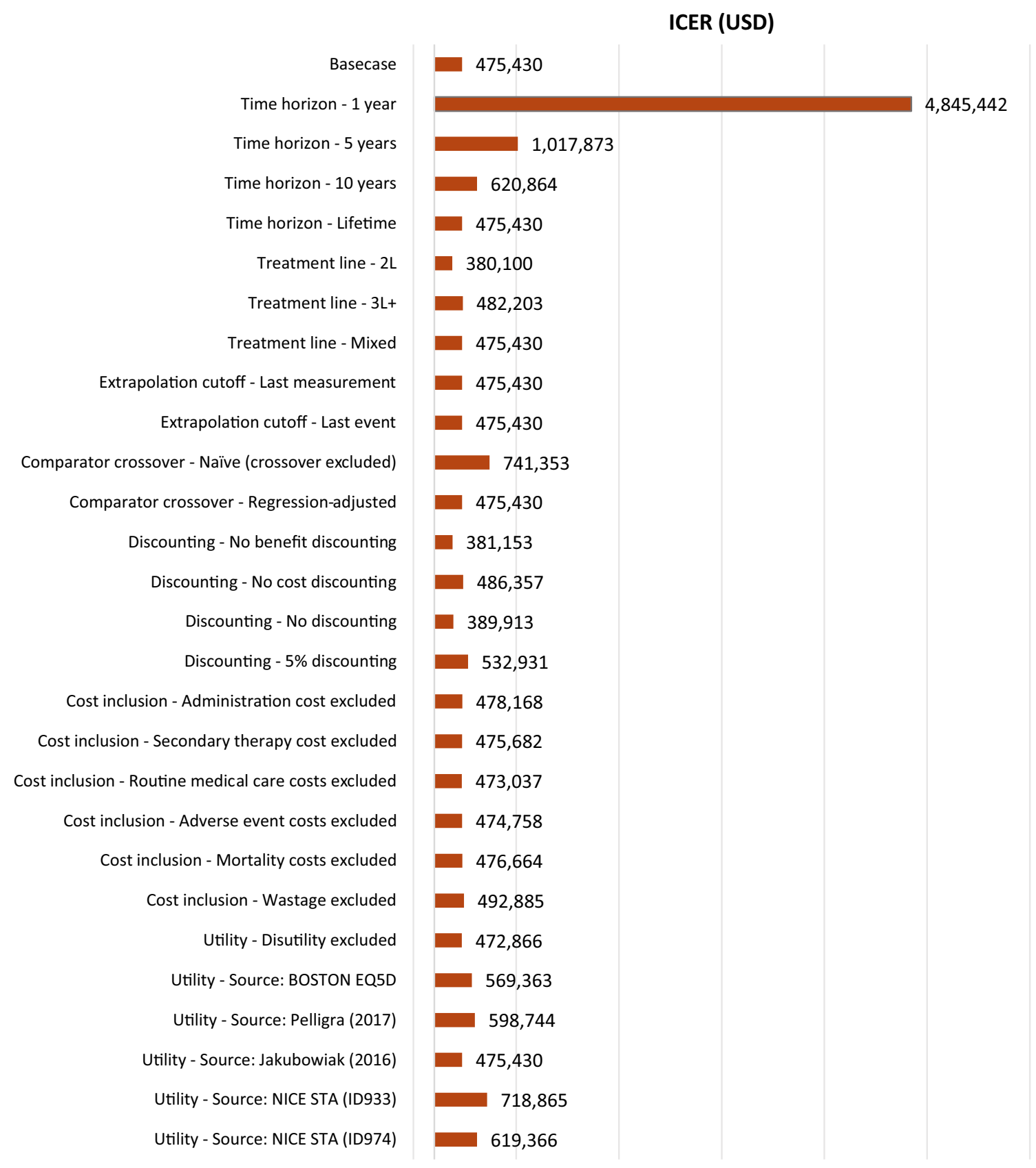

Fig. 5 Additional scenarios. $2 L$ second-line, $3 L$ third-line, ICER incremental cost-effectiveness ratio, $L$ line of therapy, $N I C E$ National Institute for Health and Care Excellence, STA single technology appraisal, USD US dollars

(contrary to several literature sources showing a greater utility difference between patients in the different states), external literature sources were used to derive the base-case utilities. While the study used in the base-case analysis had several key differences compared with the BOSTON trial population (most notably being based on UK population tariffs using data from newly diagnosed MM), the utility distinction between health states was considered to be more representative than the utility values from the BOSTON utility analysis, which showed virtually no difference between the PF and PP health states. Based on the sensitivity analyses findings, utilities were a main driver and would therefore have a significant impact on model results. Second, efficacy (PFS and OS) data had a limited follow-up duration and required long-term extrapolation, which can have a significant impact on progression and survival estimates. Additionally, no data were available to inform the duration of secondary therapy that patients would receive after discontinuing their primary therapy, thus this duration was based on assumption. Last, the model did not include the costs 
ICER (USD)

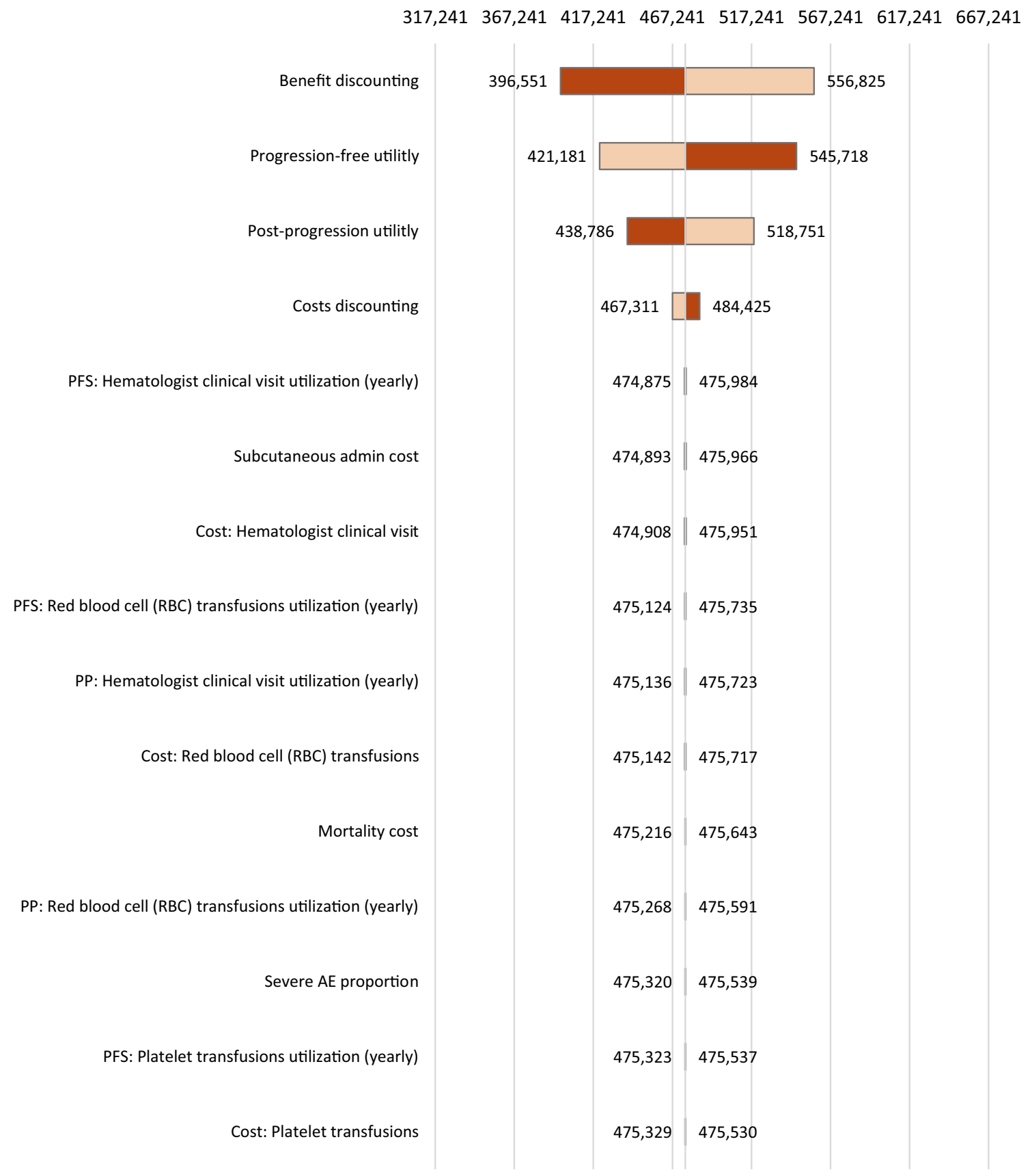

Fig. 6 Deterministic sensitivity analyses (top 15). admin administration, $A E$ adverse event, ICER incremental cost-effectiveness ratio, $P F S$ progression-free survival, $P P$ post-progression, $R B C$ red blood cell, $U S D$ US dollars

associated with lost productivity due to mortality, which may discount the calculated costs of early mortality.

Regarding the exploratory additional comparator analysis, several important limitations were present. First, some of the included regimens did not have PFS and/or OS outcomes reported in their respective clinical trials, which required significant assumptions to inform these values. Second, while many of the PFS and OS HRs were available from the clinical trials, the network meta-analysis conducted to acquire treatment line-stratified HRs was limited by the complexity of the network (which sometimes included $>20$ regimens), as well as the limited number of trials for each regimen and certain key transitivity issues in the comparison network (most notably, the requirement of a retrospective analysis 
Table 5 Probabilistic sensitivity analysis summary

\begin{tabular}{|c|c|c|c|c|c|c|c|c|}
\hline \multirow[t]{2}{*}{ Distribution } & \multicolumn{2}{|l|}{$\mathrm{XVd}$} & \multicolumn{2}{|l|}{$\mathrm{Vd}$} & \multicolumn{2}{|c|}{ Difference } & \multirow[t]{2}{*}{ Estimated ICER (\$) } & \multirow{2}{*}{$\begin{array}{l}\text { Simulated } \\
\text { ICER (\$) }\end{array}$} \\
\hline & Cost $(\$)$ & QALYs & Cost $(\$)$ & QALYs & Cost $(\$)$ & QALYs & & \\
\hline Average & 382,533 & 3.11 & 222,948 & 2.77 & 159,585 & 0.34 & 475,965 & \\
\hline $5 \%$ & 360,760 & 3.40 & 202,570 & 3.03 & 156,927 & 0.28 & 568,549 & 397,290 \\
\hline $25 \%$ & 373,408 & 3.23 & 214,506 & 2.88 & 158,622 & 0.31 & 505,317 & 435,804 \\
\hline $50 \%$ & 382,873 & 3.12 & 222,625 & 2.77 & 159,798 & 0.34 & 470,241 & 469,292 \\
\hline $75 \%$ & 390,991 & 2.99 & 230,890 & 2.66 & 160,685 & 0.37 & 438,557 & 508,027 \\
\hline $95 \%$ & 404,979 & 2.81 & 245,124 & 2.51 & 161,664 & 0.40 & 401,205 & 577,572 \\
\hline
\end{tabular}

$\$$ US dollars, ICER incremental cost-effectiveness ratio, $Q A L Y$ quality-adjusted life-year, $V d$ bortezomibdexamethasone, $X V d$ selinexor-bortezomib-dexamethasone

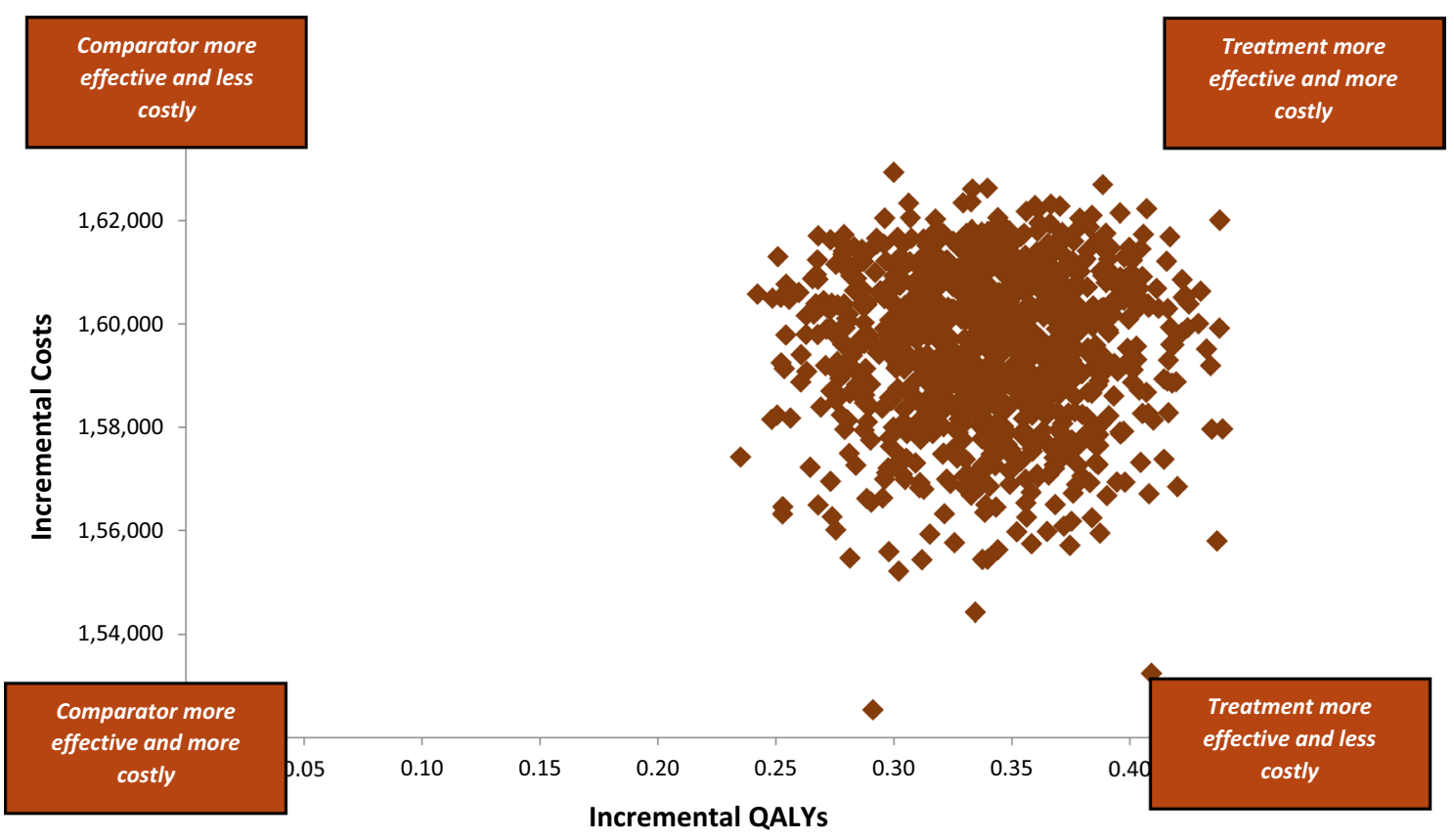

Fig. 7 Cost-effectiveness plane. $Q A L Y$ quality-adjusted life-year

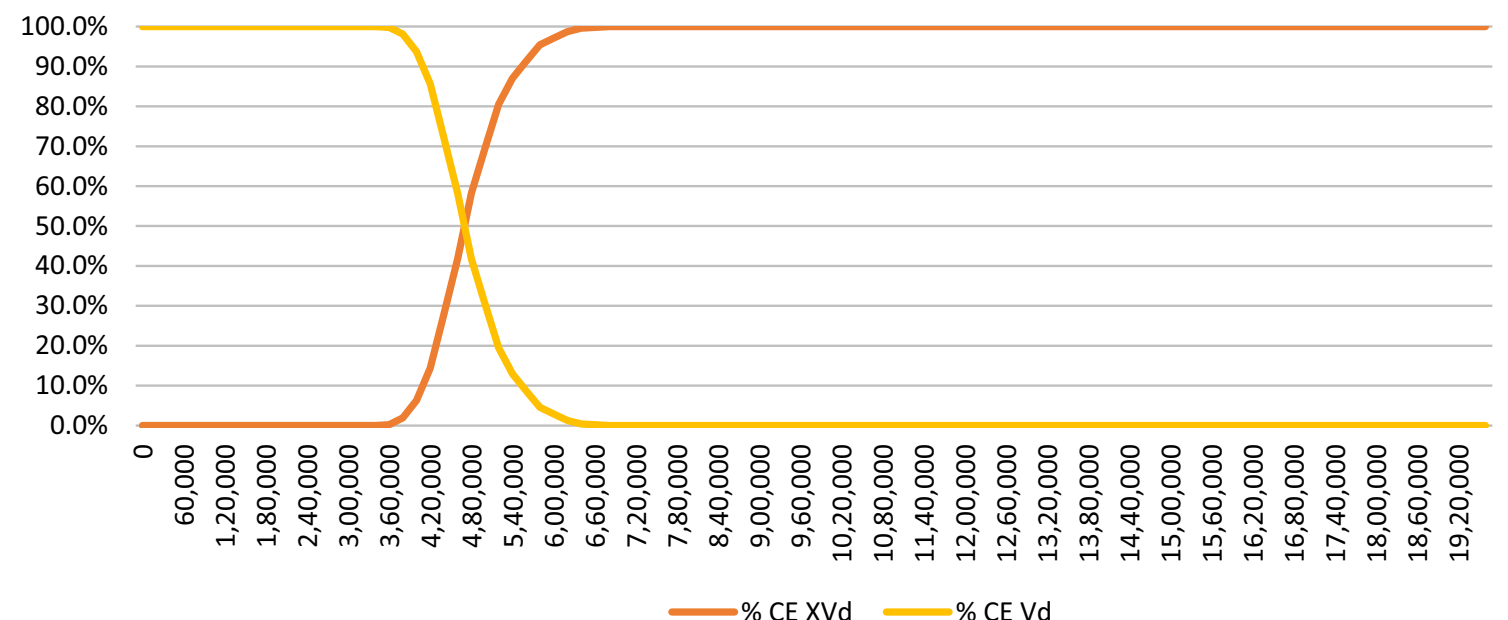

Fig. 8 Net benefit analysis. $C E$ cost effective, $V d$ bortezomib-dexamethasone, $X V d$ selinexor-bortezomib-dexamethasone 


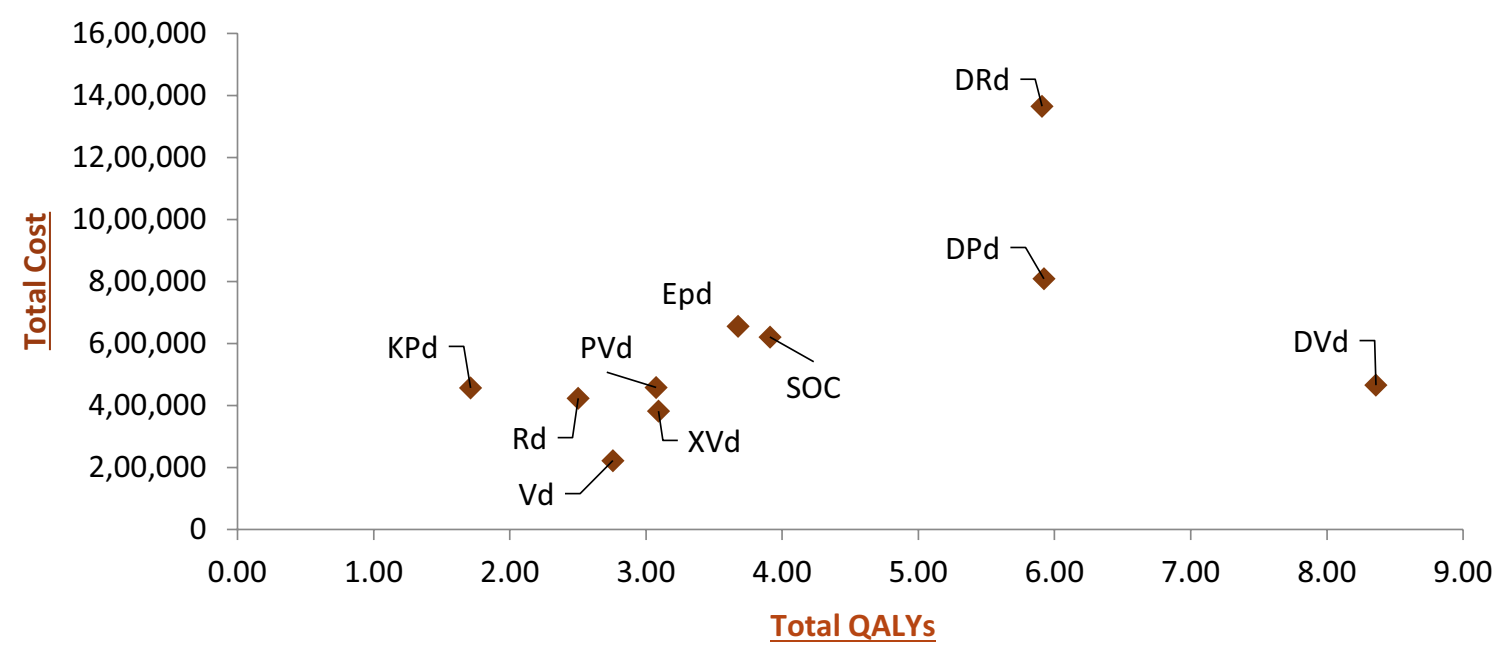

Fig. 9 Comparator analysis plot (incremental cost and qualityadjusted life-year [QALY] of selinexor-bortezomib-dexamethasone [XVd] vs comparator). DPd daratumumab-pomalidomide-dexamethasone, $D R d$ daratumumab-lenalidomide-dexamethasone, $D V d$ daratu- mumab-bortezomib-dexamethasone, $E P d$ elotuzumab-pomalidomidedexamethasone, $K P d$ carfilizomib-pomalidomide-dexamethasone, $P V d$ pomalidomide-bortezomib-dexamethasone, $R d$ lenalidomidedexamethasone, $S O C$ standard of care, $V d$ bortezomib-dexamethasone

\section{Declarations}

Further studies comparing link between some of the comparators). availability of data, and additional indirect matching techniques (matching-adjusted indirect comparison, simulated treatment comparison) could potentially add robustness to these comparisons.

\section{Conclusions}

Despite improvement in treatments, nearly all patients with MM relapse following therapy and progress to multiple treatments. This CEA describes once-weekly XVd vs twiceweekly $\mathrm{Vd}$ in the treatment of previously treated MM from a US commercial payer perspective (including additional administration-related costs) over a lifetime horizon. The base-case analysis comparing XVd to Vd estimated an ICER of $\$ 475,430$ per QALY. Deterministic sensitivity analyses and PSAs generally demonstrated little variance; supporting the robustness of the analysis. Exploratory analyses comparing $\mathrm{XVd}$ to other available regimens showed $\mathrm{XVd}$ as dominant, with a lower cost and a higher QALY gain, when compared with $\mathrm{Rd}, \mathrm{PVd}$, and $\mathrm{KPd}$. The addition of $\mathrm{XVd}$ to the treatment landscape for previously treated MM provides a novel oral regimen.

Supplementary Information The online version contains supplementary material available at https://doi.org/10.1007/s40273-021-01068-9.

Acknowledgments Dorothy Cooperson Vieweg of Purple Squirrel Economics provided manuscript writing support, funded by Karyopharm Therapeutics.
Funding Funding was provided by Karyopharm Therapeutics.

Conflicts of interest/Competing interests H. Leong is an employee of Karyopharm Therapeutics. M. Dolph and G. Tremblay are employees of Purple Squirrel Economics, which received funding from Karyop-

Ethics approval Not applicable.

Consent to participate Not applicable.

Consent for publication Not applicable.

Availability of data and material Data from the BOSTON trial can be found in "Once-per-week selinexor, bortezomib, and dexamethasone versus twice-per-week bortezomib and dexamethasone in patients with multiple myeloma (BOSTON): a randomised, open-label, phase 3 trial" by Grosicki et al. [16].

Code availability Not applicable.

Authors' contributions MD and GT were responsible for data analyses and data interpretation and participated in the drafting and revising of the manuscript. All authors were responsible for the study conception and design and participated in data interpretation and reviewed the manuscript.

Open Access This article is licensed under a Creative Commons Attribution-NonCommercial 4.0 International License, which permits any non-commercial use, sharing, adaptation, distribution and reproduction in any medium or format, as long as you give appropriate credit to the original author(s) and the source, provide a link to the Creative Commons licence, and indicate if changes were made. The images or other third party material in this article are included in the article's Creative Commons licence, unless indicated otherwise in a credit line to the material. If material is not included in the article's Creative Commons harm Therapeutics to conduct this study. 
licence and your intended use is not permitted by statutory regulation or exceeds the permitted use, you will need to obtain permission directly from the copyright holder. To view a copy of this licence, visit http://creativecommons.org/licenses/by-nc/4.0/.

\section{References}

1. American Cancer Society. Key statistics for multiple myeloma. 2021. https://www.cancer.org/cancer/multiple-myeloma/about/ key-statistics.html. Accessed 22 Jul 2021.

2. Merola D, Yong C, Noga SJ, Shermock KM. Costs associated with productivity loss among U.S. patients newly diagnosed with multiple myeloma receiving oral versus injectable chemotherapy. J Manag Care Spec Pharm. 2018;24:1019-26.

3. Chari A, Romanus D, DasMahapatra P, Hoole M, Lowe M, Curran $\mathrm{C}$, et al. Patient-reported factors in treatment satisfaction in patients with relapsed/refractory multiple myeloma (RRMM). Oncologist. 2019;24:1479-87.

4. Fonseca R, Abouzaid S, Bonafede M, Cai Q, Parikh K, Cosler L, et al. Trends in overall survival and costs of multiple myeloma, 2000-2014. Leukemia. 2017;31:1915-21.

5. MacEwan JP, Batt K, Yin W, Peneva D, Sison S, Vine S, et al. Economic burden of multiple myeloma among patients in successive lines of therapy in the United States. Leuk Lymphoma. 2018;59:941-9.

6. Leleu X, Martin TG, Einsele H, Lyons RM, Durie BGM, Iskander KS, et al. Role of proteasome inhibitors in relapsed and/ or refractory multiple myeloma. Clin Lymph Myeloma Leuk. 2019;19:9-22.

7. Millenium Pharmaceuticals Inc. Velcade $®$ (bortezomib) [prescribing information]. 2019. https://www.velcade.com/files/pdfs/ VELCADE_PRESCRIBING_INFORMATION.pdf. Accessed 22 Jul 2021

8. Dimopoulos MA, Moreau P, Palumbo A, Joshua D, Pour L, Hájek $\mathrm{R}$, et al. Carfilzomib and dexamethasone versus bortezomib and dexamethasone for patients with relapsed or refractory multiple myeloma (ENDEAVOR): a randomised, phase 3, open-label, multicentre study. Lancet Oncol. 2016;17:27-38.

9. Grammatico S, Petrucci M, Cesini L. Managing treatment-related peripheral neuropathy in patients with multiple myeloma. Blood Lymphat Cancer Targets Ther. 2016;6:37-47.

10. Palumbo A, Chanan-Khan A, Weisel K, Nooka AK, Masszi T, Beksac M, et al. Daratumumab, bortezomib, and dexamethasone for multiple myeloma. N Engl J Med. 2016;375:754-66.

11. Richardson PG, Oriol A, Beksac M, Liberati AM, Galli M, Schjesvold F, et al. Pomalidomide, bortezomib, and dexamethasone for patients with relapsed or refractory multiple myeloma previously treated with lenalidomide (OPTIMISMM): a randomised, openlabel, phase 3 trial. Lancet Oncol. 2019;20:781-94.

12. Hu B, Zhou Q, Hu Y, Zhuang L, Yi L, Cao J, et al. Efficacy and safety of once-weekly versus twice-weekly bortezomib in patients with hematologic malignancies: a meta-analysis with trial sequential analysis. Pharmacotherapy. 2019;39:697-708.

13. Bringhen S, Larocca A, Gugielmelli T, Benevolo G, Callea V, Cangialosi C, et al. Efficacy and safety of once-weekly bortezomib in multiple myeloma patients. Blood. 2010;116(23):4745-53.

14. Richardson PG, Sonneveld P, San Miguel J, Blade J, Boccadoro $\mathrm{M}$, Cavenagh J, et al. Extended follow-up of a phase 3 trial in relapsed multiple myeloma : final time-to-event results of the APEX trial. 2007. http://pascal-francis.inist.fr/vibad/index.php? action $=$ search\&terms $=19690810$. Accessed 22 Jul 2021.

15. Xpovio USPI. Xpovio ${ }^{\mathrm{TM}}$ (selinexor) tablets [prescribing onformation]. Newton: Karyopharm Therapeutics, Inc.; 2020.
16. Grosicki S, Simonova M, Spicka I, Pour L, Kriachok I, Gavriatopoulou M, et al. Once-per-week selinexor, bortezomib, and dexamethasone versus twice-per-week bortezomib and dexamethasone in patients with multiple myeloma (BOSTON): a randomised, open-label, phase 3 trial. Lancet. 2020;396:1563-73.

17. Sanchez L, LeLeu X, Beaumont J. Peripheral neuropathy symptoms, pain and functioning in relapsed or refractory multiple myeloma patients treated with selinexor, bortezomib, and dexamethasone [abstract]. ASH; 2020. https://ash.confex.com/ash/ 2020/webprogram/Paper141319.html. Accessed 3 Dec 2020.

18. Karyopharm Therapeutics Inc. Clinical study report Kcp-330-023: a phase 3 randomized, controlled, open-label study of selinexor, bortezomib, and dexamethasone (SVd) versus bortezomib and dexamethasone $(\mathrm{Vd})$ in patients with relapsed or refractory multiple myeloma (RRMM). 2020.

19. Sanders GD, Neumann PJ, Basu A, Brock DW, Feeny D, Krahn $\mathrm{M}$, et al. Recommendations for conduct, methodological practices, and reporting of cost-effectiveness analyses: Second Panel on Cost-Effectiveness in Health and Medicine. JAMA. 2016;316:1093-103.

20. Microsoft Corporation. Excel. 2021. https://office.microsoft.com/ excel. Accessed 22 Jul 2021.

21. United Stated Bureau of Labor Statistics. CPI disease based price index (DBI): neoplasm diseases. 2020. Report No.: 20162020. https://www.bls.gov/pir/diseasehome.htm. Accessed 22 Jul 2021.

22. American Medical Association. 2020 final Medicare coding \& payment for drug administration services under the hospital outpatient prospective payment system. Johnson \& Johnson Health Care Systems Inc.; 2019. https://www.janssencarepath.com/sites/www. janssencarepath.com/files/medicare-coding-and-payment-drugadmin-services-hospital-outpatient.pdf. Accessed 22 Jul 2021.

23. Karyopharm. BOSTON clinical trial: adqs data set. 2020.

24. Mateos M-V, Usmani SZ, Grosicki S, Vorobyev VI, Spicka I, Hungria VTM, et al. Randomized, open-label, non-inferiority, phase 3 study of subcutaneous (SC) versus intravenous (IV) daratumumab (DARA) administration in patients (Pts) with relapsed or refractory multiple myeloma (RRMM): body weight subgroup analysis of Columba. Blood. 2019;134:1906.

25. Analy\$ource. AWP drug pricing. 2020. https://www.analysource. com. Accessed 21 Oct 2020.

26. Centers for Medicare \& Medicaid Services. Medical care costs. 2020. https://www.cms.gov/. Accessed 11 Oct 2020.

27. Agency for Healthcare Research and Quality. 2017 hospital charges. 2020. https://hcupnet.ahrq.gov. Accessed 20 Oct 2020.

28. Pelligra CG, Parikh K, Guo S, Chandler C, Mouro J, Abouzaid $\mathrm{S}$, et al. Cost-effectiveness of pomalidomide, carfilzomib, and daratumumab for the treatment of patients with heavily pretreated relapsed-refractory multiple myeloma in the United States. Clin Ther. 2017;39:1986-2005.e5.

29. Fragoulakis V, Kastritis E, Psaltopoulou T, Maniadakis N. Economic evaluation of therapies for patients suffering from relapsedrefractory multiple myeloma in Greece. Cancer Manag Res. 2013;5:37-48.

30. Agency for Healthcare Research and Quality. Mortality costs. 2020. https://hcupnet.ahrq.gov. Accessed 11 Oct 2020.

31. van Agthoven M, Segeren CM, Buijt I, Uyl-de Groot CA, van der Holt B, Lokhorst HM, et al. A cost-utility analysis comparing intensive chemotherapy alone to intensive chemotherapy followed by myeloablative chemotherapy with autologous stemcell rescue in newly diagnosed patients with stage II/III multiple myeloma; a prospective randomised phase III study. Eur J Cancer. 2004:40:1159-69.

32. Institute for Clinical and Economic Review. ICER 2020-2023 value assessment framework. 2020. http://icer.org/wp-content/ uploads/2020/10/ICER_2020_2023_VAF_102220.pdf. Accessed 22 Oct 2020. 
33. Caro JJ, Briggs AH, Siebert U, Kuntz KM, ISPOR-SMDM Modeling Good Research Practices Task Force. Modeling good research practices-overview: a report of the ISPOR-SMDM Modeling Good Research Practices Task Force-1. Value Health. 2012;15:796-803.

34. Patel K, Parker TL, Di M. Cost-effectiveness of once-weekly selinexor, bortezomib, and dexamethasone ( $\mathrm{SVd}$ ) versus twiceweekly bortezomib and dexamethasone (Vd) in relapsed or refractory multiple myeloma. 2020. https://ash.confex.com/ash/2020/ webprogram/Paper141972.html. Accessed 11 Dec 2020.

35. Asrar MM, Lad DP, Prinja S, Bansal D. A systematic review of economic evaluations of treatment regimens in multiple myeloma. Expert Rev Pharmacoecon Outcomes Res. 2020; p. 1-11.

36. Carlson JJ, Guzauskas GF, Chapman RH, Synnott PG, Liu S, Russo ET, et al. Cost-effectiveness of drugs to treat relapsed/ refractory multiple myeloma in the United States. J Manag Care Spec Pharm. 2018;24:29-38.

37. Gong CL, Studdert AL, Liedtke M. Daratumumab vs. pomalidomide for the treatment of relapsed/refractory multiple myeloma: a cost-effectiveness analysis. Am J Hematol. 2019;94:E68-70.

38. Pelligra C, Guo S, Parikh K, Zhang S, Krotneva M, Onyekwere $\mathrm{U}$, et al. Cost-effectiveness analysis of daratumumab, lenalidomide, and dexamethasone (DRD) and daratumumab, bortezomib, and dexamethasone (DVD) versus standard of care in relapsed or refractory multiple myeloma (RRMM). Value Health. 2018;21:S254. 